\title{
Elaboración de recubrimientos cerámicos mediante proyección térmica por combustión a partir de residuos sólidos industriales
}

\author{
Elaboration of ceramic coatings by flame spraying \\ process from industrial solid waste
}

\author{
Restrepo, E. ${ }^{2}$; Vargas, F. ${ }^{2}$; López, M. ${ }^{2}$; \\ Cardona, R. ${ }^{2}$; Duarte, G. ${ }^{1}$
}

\author{
${ }^{1}$ Instituto Colombiano del Petróleo, ICP-ECOPETROL, c.p. 4185, Piedecuesta, Colombia. \\ e-mail:gloriai.duarte@ecopetrol.com.co \\ ${ }^{2}$ Grupo GIPIMME-GIMACYR, Universidad de Antioquia, Medellín, Colombia. \\ e-mail: edward.restrepo@yahoo.es ; vargasf@udea.edu.co; melopez@udea.edu.co ; lordroger_64@hotmail.com
}

\section{RESUMEN}

Mediante la tecnología de proyección térmica por combustión oxiacetilénica se aplicaron sobre sustratos de acero al carbono AISI-SAE 1020 recubrimientos cerámicos a partir de residuos de catalizadores utilizados en procesos de refinación en la industria del petróleo. La adherencia y la dureza de los recubrimientos fueron evaluadas según las normas C633-01(2008) y ASTM C1327-08 respectivamente, con valores de adhesión que oscilan entre 2,9 y 17,7 MPa y valores de microdureza Vickers entre 5,4 y 8 GPa.

Los resultados obtenidos permiten visualizar su posible aplicación industrial en componentes que requieran resistencia al desgaste, a las altas temperaturas o al choque térmico, a un costo menor que los elaborados con materias primas comerciales, debido a la re-utilización de los residuos sólidos en la elaboración de los recubrimientos y generando valor agregado como solución tecnológica al impacto ambientalmente negativo que generan los residuos de los catalizadores.

Palabras claves: Recubrimientos cerámicos, proyección térmica, residuos de refinación.

\section{ABSTRACT}

Ceramic coatings were elaborated onto carbon steel substrates by oxy-fuel thermal spraying process from catalysts waste used in petroleum refining. The adhesion and micro-hardness Vickers were evaluated according to ASTM C633-01(2008) and ASTM C1327-08 standards respectively, with adhesion values varying between 2,9 and 17,7 MPa and values of microhardness Vickers between 5,4 and $8 \mathrm{GPa}$. The results obtained indicate that these coatings could be used in industrial applications, mainly in components requiring resistance to wear and thermal shock or high temperatures, at a low cost due to reuse of solid waste as raw materials in the production of coatings, and giving a technological solution to environment problems produced by these catalysts waste.

Keywords: Ceramic coatings, thermal spray, catalysts waste. 


\section{INTRODUCCIÓN}

Durante la refinación del petróleo los catalizadores juegan un papel fundamental en la conversión de las fracciones pesadas del crudo en fracciones más ligeras. Estos catalizadores están compuestos principalmente por materiales cerámicos (óxidos metálicos), los cuales debido a la pérdida de las propiedades catalíticas, producidas por el ensuciamiento, envejecimiento y/o pérdida de la fase activa por volatilización, son remplazados al cumplir su ciclo de vida útil, los catalizadores en esta etapa son conocidos como catalizadores gastados [1-5].

En la industria petroquímica se generan grandes cantidades de catalizadores gastados, cantidad que en la actualidad incrementa continuamente debido al aumento en la demanda de combustibles, lo cual conlleva a una mayor problemática ambiental por la disposición final de los mismos. En Colombia, el manejo de los catalizadores gastados, representa un problema ambiental que afecta el entorno y la calidad de vida de la población, producto de su disposición final $[3, \underline{6}, 7]$. Típicamente los catalizadores gastados son reutilizados como material de relleno en la industria cementera para la fabricación de hormigón y en la producción de ladrillos [6].

Teniendo en cuenta que los residuos de los catalizadores tienen una morfología, distribución granulométrica y composición química similar a los polvos comerciales utilizados para la producción de recubrimientos cerámicos, que en su mayoría están compuestos por alúmina $\left(\mathrm{Al}_{2} \mathrm{O}_{3}\right)$ y alúmina-sílice $\left(\mathrm{Al}_{2} \mathrm{O}_{3^{-}}\right.$ $\mathrm{SiO}_{2}$ ), éstos fueron tratados y re-utilizados como materia prima para la elaboración de recubrimientos cerámicos mediante proyección térmica por combustión oxi-acetilénica, sobre sustratos de acero al carbono AISI-SAE 1020. Las propiedades físico-mecánicas, dureza y adherencia, de los recubrimientos obtenidos son similares a las de los elaborados a partir de polvos comerciales; logrando de esta forma dar valor agregado a estos residuos industriales, disminuyendo el costo en la elaboración de los recubrimientos y ofreciendo una solución tecnológica al impacto ambiental negativo que generan los residuos de los catalizadores de refinación.

Adicionalmente, el proceso de proyección térmica por combustión oxiacetilénica permite recubrir piezas de grandes dimensiones y diversas geometrías gracias a la versatilidad del equipo, siendo además un proceso económico, lo cual viabiliza su utilización en la aplicación de recubrimientos para diversos componentes industriales $[8,9]$.

\section{DESARROLLO EXPERIMENTAL}

Se utilizaron tres residuos de catalizadores gastados para su reconversión en materia prima para la elaboración de los recubrimientos cerámicos mediante proyección térmica, los cuales fueron codificados como $\mathrm{M}_{1}, \mathrm{M}_{2}$ y $\mathrm{M}_{3}$. En la primera etapa se realizó un muestreo utilizando el método manual de cono y cuarteo de acuerdo con la norma ASTM C702/C702M-11, con el objetivo de seleccionar una muestra representativa de cada uno de los residuos a procesar; posteriormente se realizó la caracterización fisicoquímica de las muestras seleccionadas.

\subsection{Caracterización fisicoquímica de los residuos}

Se realizó análisis químico mediante Fluorescencia de Rayos X por longitud de onda dispersiva utilizando el equipo ARL OPTIM'X-211, y se determinaron las fases presentes en las muestras de los catalizadores mediante difracción de rayos X utilizando el equipo Panalitycal X'Pert Pro MPD y la base de datos X'Pert High Score. Las características físicas de las partículas, tamaño y forma, fueron determinadas por análisis granulométrico por difracción láser empleando el equipo Máster Sizer 2000, y por microscopia electrónica de barrido (MEB), utilizando el equipo JEOL JSM 6490 LV.

\subsection{Procesamiento de los catalizadores}

El acondicionamiento del tamaño de partícula de los catalizadores gastados para su utilización como materia 
prima en la elaboración de recubrimientos mediante proyección térmica, se realizó mediante clasificación mecánica.

\subsection{Elaboración de los recubrimientos}

Para la elaboración de los recubrimientos se utilizó la cámara Areste-1 de la Universidad de Antioquia, Figura 1 (a), la cual está provista de la antorcha Terodyn 2000, de controles electro-mecánicos y de un pirómetro óptico; que permiten sensar, controlar y registrar los parámetros de proyección, tales como la temperatura del sustrato y del recubrimiento durante el proceso, la velocidad relativa entre la antorcha y el sustrato y la distancia de proyección, entre otras. Las condiciones de aplicación de los recubrimientos fueron establecidas con base en los resultados de estudios preliminares y con la ayuda del Software Jets et Poudres, Figura 1 (b), el cual permite simular procesos de proyección térmica por combustión. Previo a la elaboración de los recubrimientos los sustratos de acero al carbono AISI-SAE 1020 fueron preparados superficialmente mediante chorro abrasivo de corindón, con posterior lavado por ultrasonido en alcohol, con el fin de generar la rugosidad y limpieza necesarias para el anclaje de las partículas proyectadas.

La distancia de proyección entre el sustrato y la antorcha fue de $7 \mathrm{~cm}$ con un rango de temperatura de precalentamiento de la superficie entre $112{ }^{\circ} \mathrm{C}$ y $150{ }^{\circ} \mathrm{C}$. El flujo de oxigeno fue de $37 \mathrm{l} / \mathrm{min}$ y el de acetileno de $22 \mathrm{l} / \mathrm{min}$, y la presión del jet de oxígeno utilizado para propulsar las partículas hacia el sustrato fue de 30 psi.
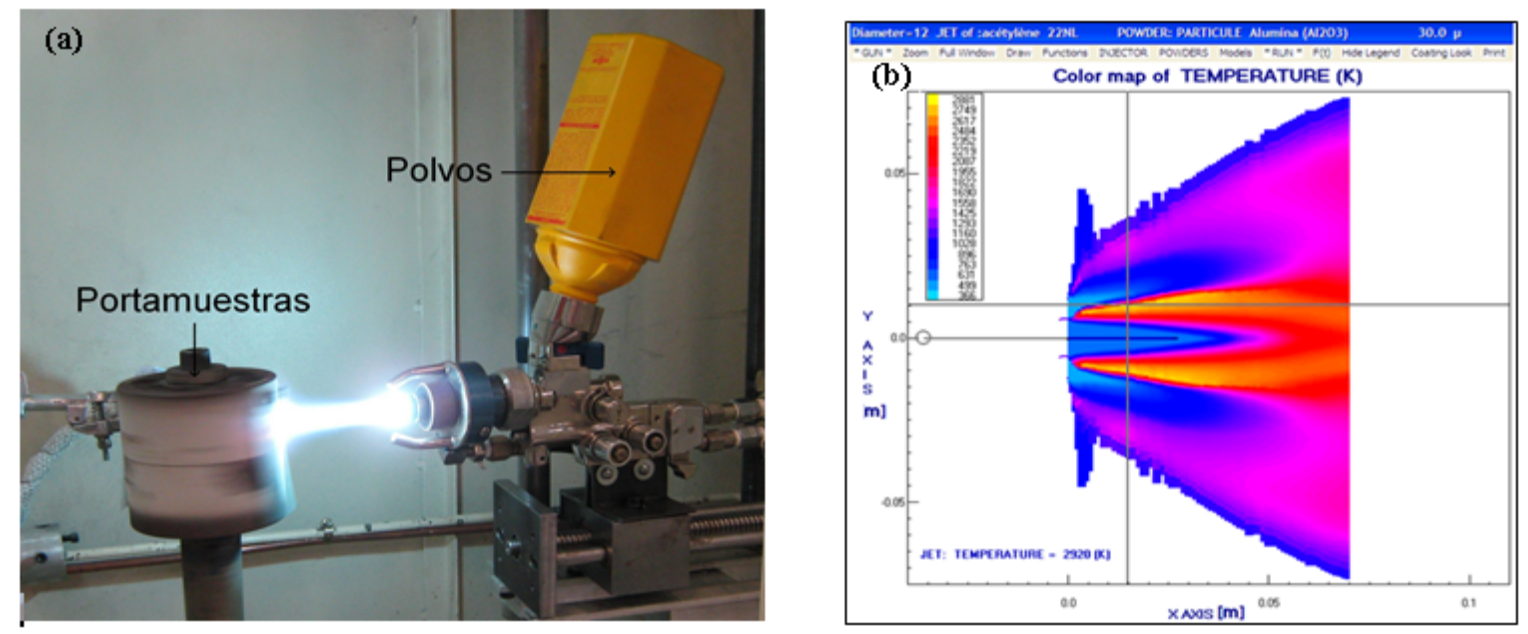

Figura 1: (a) Cámara Areste-1, (b) resultados de los perfiles de temperatura de la llama oxi-acetilénica obtenidos por simulación mediante el Software Jets et Poudres.

\subsection{Caracterización de los recubrimientos}

La caracterización estructural de los recubrimientos se realizó mediante el análisis de su sección transversal, empleando microscopía electrónica de barrido (MEB), para lo cual fue utilizado un equipo JEOL JSM 6490 LV. Para tal efecto, las muestras fueron preparadas según los lineamientos de la norma ASTM E192003(2008). Adicionalmente se determinaron las fases presentes mediante difracción de rayos X, utilizando el equipo Panalitycal X'Pert Pro MPD y la base de datos X'Pert High Score. La dureza de los recubrimientos fue determinada sobre la sección transversal mediante indentación Vickers aplicando una carga de 200 gramos durante 15 segundos y teniendo en cuenta los lineamientos de la norma ASTM C1327-08; mientras que, la adherencia entre los recubrimientos depositados y el sustrato fue determinada mediante ensayo de tensión, según las especificaciones de la norma ASTM C633-01(2008) utilizando un adhesivo tipo epoxi. 


\section{RESULTADOS Y ANÁLISIS DE RESULTADOS}

\subsection{Caracterización de los residuos de los catalizadores}

\subsubsection{Muestra $M_{1}$}

En la Tabla 1, se muestran los resultados del análisis químico realizado a la muestra $\mathrm{M}_{1}$, la cual está constituida por $99,75 \%$ en peso de $\mathrm{Al}_{2} \mathrm{O}_{3} ; 0,16 \%$ en peso de $\mathrm{SiO}_{2} ;$ y $0,09 \%$ en peso de otros óxidos. Esta muestra posee una morfología irregular y su interior es poroso, Figura 2. La distribución de tamaño de partícula es bimodal, con un pico entre 1 y $85 \mu \mathrm{m}$ y el otro entre 85 y $1000 \mu \mathrm{m}$, y varía entre 0,5 y $1000 \mu \mathrm{m}$, como se observa en la Figura 3 (a). Las fases cristalinas presentes son Bohemita $\left(\mathrm{AlHO}_{2}\right.$ ortorrómbico) y Óxido de Aluminio $\left(\mathrm{Al}_{2} \mathrm{O}_{4}\right.$ ortorrómbico), Figura $3(\mathrm{~b})$.

Tabla 1: Resultados del análisis químico realizado a la muestra $\mathrm{M}_{1}$.

\begin{tabular}{c|c}
\hline COMPUESTO & ANÁLISIS QUÍMICO \% EN PESO \\
\hline $\mathrm{Al}_{2} \mathrm{O}_{3}$ & $99,75 \pm 0,03$ \\
\hline $\mathrm{SiO}_{2}$ & $0,16 \pm 0,02$ \\
\hline Otros óxidos & $0,09 \pm 0,02$ \\
\hline
\end{tabular}
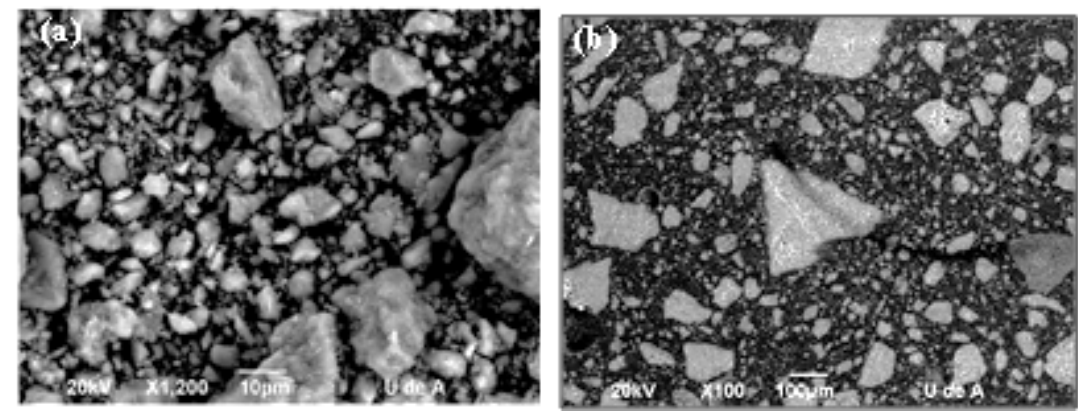

Figura 2: Morfología de las partículas de la muestra $\mathrm{M}_{1}$. (a) Superficie, (b) Sección transversal.
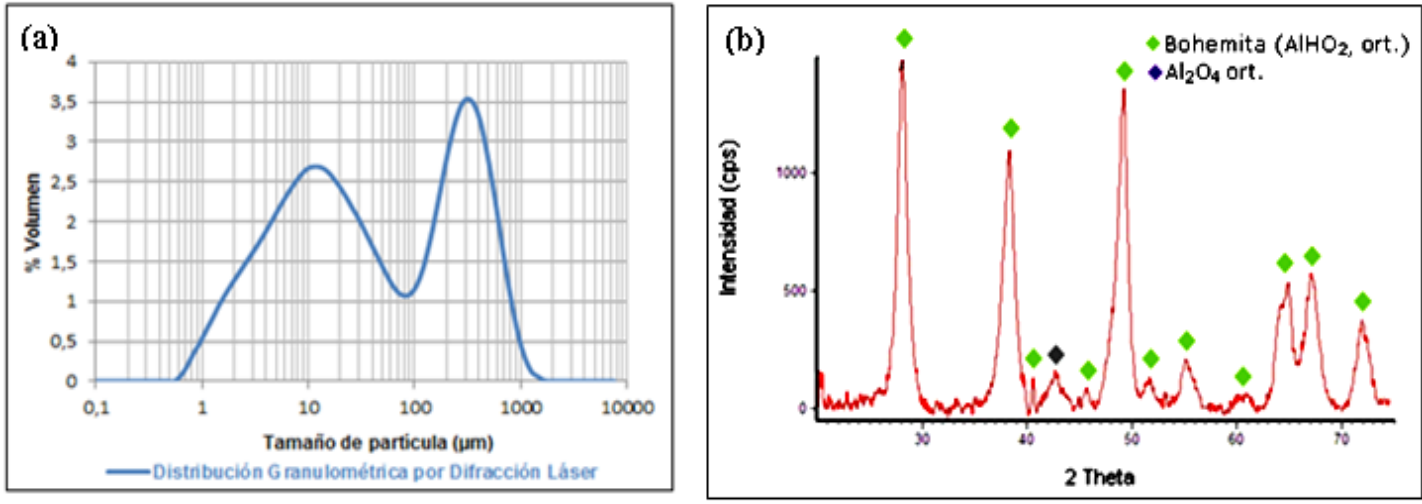

Figura 3: Muestra $\mathrm{M}_{1}$, (a) Distribución del tamaño de partícula, (b) Espectro de difracción de rayos X.

\subsubsection{Muestra $\mathrm{M}_{2}$}

Según los resultados del análisis químico presentado en la Tabla 2, la muestra $\mathrm{M}_{2}$ está constituida en un $94,68 \%$ en peso por $\mathrm{Al}_{2} \mathrm{O}_{3} ; 4,24 \%$ en peso de $\mathrm{SO}_{3} ; 0,83 \%$ en peso de $\mathrm{TiO}_{2} ;$ y $0,11 \%$ en peso de $\mathrm{SiO}_{2}$, el 
porcentaje restante corresponde a otros óxidos. Esta muestra posee una morfología irregular y las partículas en su interior son porosas, Figura 4. La distribución de tamaño de partícula varía entre 0,5 y $1000 \mu \mathrm{m}$, con dos picos, el primero entre 0,5 y $100 \mu \mathrm{m}$, y el segundo entre 100 y $800 \mu \mathrm{m}$, como se observa en la Figura 5 (a). Las fases cristalinas presentes en esta muestra son alúmina hexagonal $\left(\mathrm{Al}_{2} \mathrm{O}_{3}-\alpha\right)$ y sílice $\left(\mathrm{SiO}_{2}\right.$ monoclínica), Figura 5 (b).

Tabla 2: Resultados del análisis químico realizado a la muestra $\mathrm{M}_{2}$.

\begin{tabular}{c|c}
\hline COMPUESTO & ANÁLISIS QUÍMICO \% EN PESO \\
\hline $\mathrm{Al}_{2} \mathrm{O}_{3}$ & $94,68 \pm 0,11$ \\
\hline $\mathrm{SO}_{3}$ & $4,24 \pm 0,10$ \\
\hline $\mathrm{TiO}_{2}$ & $0,83 \pm 0,04$ \\
\hline $\mathrm{SiO}_{2}$ & $0,11 \pm 0,02$ \\
\hline Otros óxidos & $0,14 \pm 0,02$ \\
\hline
\end{tabular}
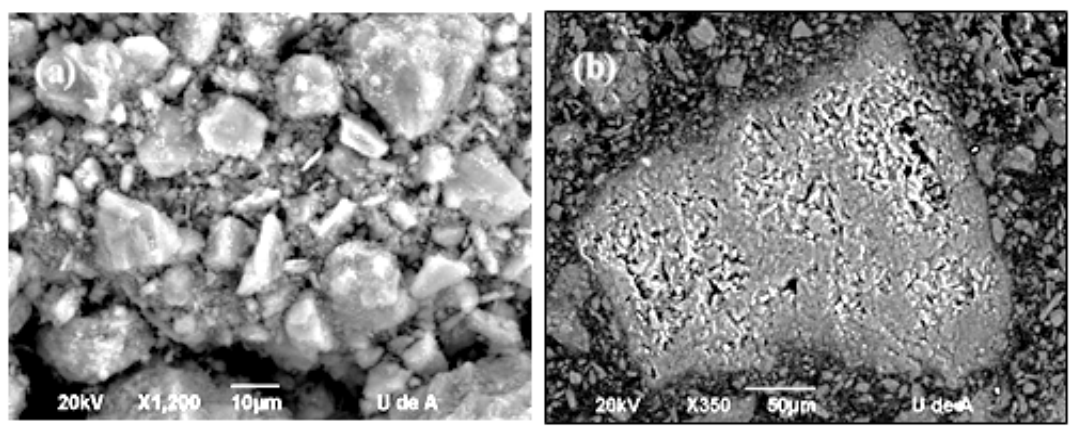

Figura 4: Morfología de las partículas de la muestra $\mathrm{M}_{2}$. (a) Superficie, (b) Sección transversal.
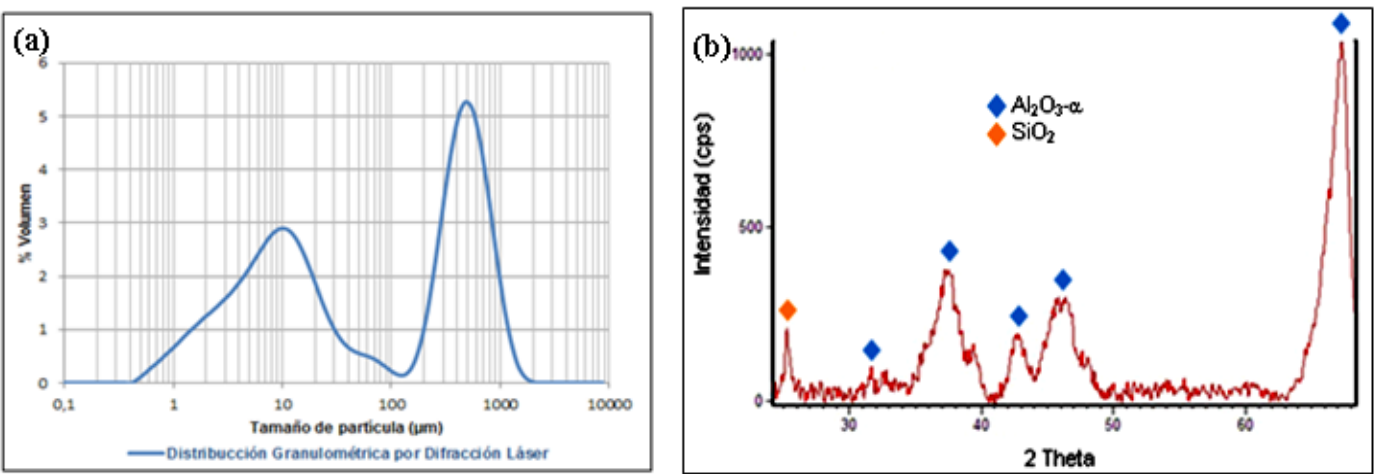

Figura 5: Muestra $\mathrm{M}_{2}$. (a) Distribución del tamaño de partícula, (b) Espectro de difracción de rayos X.

El óxido de azufre ( $\left.\mathrm{SO}_{3}\right)$, que se encuentra presente en esta muestra en un 4,24\% en peso, es altamente nocivo para el medio ambiente, ya que es uno de los causantes de la lluvia ácida y en presencia de agua reacciona violentamente dando lugar a la formación de ácido sulfúrico, siendo altamente corrosivo. No obstante, este tipo de compuesto puede ser eliminado de los catalizadores gastados mediante limpieza con etanol, benceno y disulfuro de carbono $\left(\mathrm{CS}_{2}\right)$ [10], o pueden descomponerse a dióxido de azufre $\left(\mathrm{SO}_{2}\right)$ por calentamiento, de forma similar a lo que sucede con las partículas en la llama oxiacetilénica [11]. 


\subsubsection{Muestra $\mathrm{M}_{3}$}

En la Tabla 3, se presentan los resultados del análisis químico realizado a la muestra $\mathrm{M}_{3}$, la cual está constituida principalmente por alúmina $\left(\mathrm{Al}_{2} \mathrm{O}_{3}\right)$ y sílice $\left(\mathrm{SiO}_{2}\right)$, con un 46,36 y 42,59\% en peso, respectivamente. Esta muestra posee morfología esférica y el interior de las partículas es poroso, Figura 6. Su distribución granulométrica es monomodal y varía entre 0,4 y $120 \mu \mathrm{m}$, Figura 7 (a). Las fases cristalinas presentes en este catalizador son alúmina gama $\left(\mathrm{Al}_{2} \mathrm{O}_{3}-\gamma\right)$ y sílice en forma de cuarzo y cúbica, Figura 7 (b).

Tabla 3: Resultados del análisis químico realizado a la muestra $\mathrm{M}_{3}$.

\begin{tabular}{c|c}
\hline COMPUESTO & ANÁLISIS QUÍMICO \% EN PESO \\
\hline $\mathrm{Al}_{2} \mathrm{O}_{3}$ & $46,36 \pm 0,25$ \\
\hline $\mathrm{SiO}_{2}$ & $42,59 \pm 0,25$ \\
\hline $\mathrm{La}_{2} \mathrm{O}_{3}$ & $5,38 \pm 0,11$ \\
\hline $\mathrm{V}_{2} \mathrm{O}_{5}$ & $1,33 \pm 0,06$ \\
\hline $\mathrm{Fe}_{2} \mathrm{O}_{3}$ & $0,88 \pm 0,04$ \\
\hline $\mathrm{NiO}$ & $0,87 \pm 0,04$ \\
\hline $\mathrm{TiO}_{2}$ & $0,86 \pm 0,04$ \\
\hline Otros óxidos & $1,73 \pm 0,03$ \\
\hline
\end{tabular}
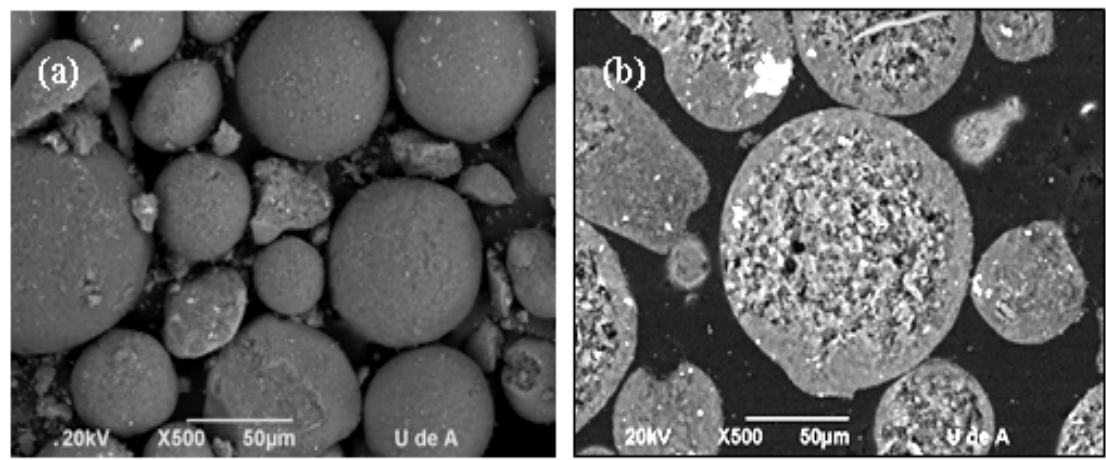

Figura 6: Morfología de las partículas de la muestra $\mathrm{M}_{3}$. (a) Superficie, (b) Sección transversal.
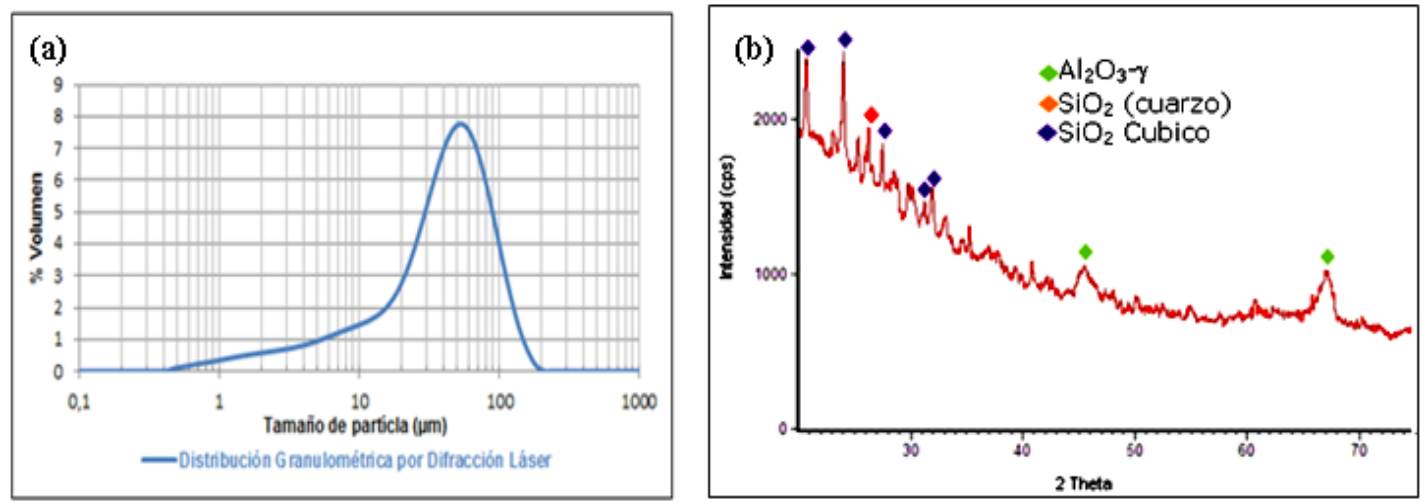

Figura 7: Muestra $\mathrm{M}_{3}$, (a) Distribución del tamaño de partícula, (b) Patrón de difracción de rayos X.

Polvos de composición química y morfología similar a los tres residuos de catalizadores evaluados son utilizados para elaborar recubrimientos mediante proyección térmica, no obstante, su tamaño de partícula es mayor al de los polvos comerciales, los cuales generalmente tienen una distribución granulométrica que oscila entre 5 - $50 \mu \mathrm{m}$ [12]. De acuerdo con lo anterior, se realizó una clasificación mecánica del tamaño de partícula a cada una de las muestras de catalizador gastado, seleccionando la fracción correspondiente a -53 
$+45 \mu \mathrm{m}$ para la elaboración del recubrimiento con la muestra $\mathrm{M}_{1}$, y la fracción correspondiente a $-45+38 \mu \mathrm{m}$ para depositar las muestras a partir del catalizador $\mathrm{M}_{2} \mathrm{y} \mathrm{M}_{3}$. La selección de la fracción de cada uno de los catalizadores se basó en su fluidez dentro del ducto que la transporta a la llama oxiacetilénica para la proyección térmica.

\subsection{Caracterización de los recubrimientos}

Los resultados de la caracterización estructural y de las fases identificadas en cada uno de los recubrimientos elaborados se presentan en las Figuras 8, 9 y 10.

El recubrimiento obtenido a partir de la muestra de catalizador gastado $\mathrm{M}_{1}$ posee una estructura constituida por partículas bien apiladas, lo cual indica que la fracción de tamaño utilizada para su elaboración es apropiada y permite obtener buena fusión de estas partículas en la llama oxiacetilénica, Figura 8 (a). También, se aprecian micro-grietas horizontales las cuales se producen principalmente por el calentamiento excesivo de las partículas durante la formación de la capa de recubrimiento. En la interfaz se observa que las partículas depositadas se acoplan bien a las irregularidades de la superficie del sustrato, lo cual favorece su adherencia. Las fases presentes en este recubrimiento son $\mathrm{Al}_{2} \mathrm{O}_{3}-\alpha$ y $\mathrm{Al}_{2} \mathrm{O}_{3}-\gamma$, como se muestra en la Figura 8 (b). Es importante resaltar que los materiales constituidos por $\mathrm{Al}_{2} \mathrm{O}_{3}-\alpha$ son ampliamente utilizados a nivel industrial, gracias a su alta dureza y estabilidad térmica en altas temperaturas.

La estructura del recubrimiento elaborado a partir del catalizador $\mathrm{M}_{2}$, Figura 9 (a), posee una cantidad importante de espacios vacíos y de micro-grietas horizontales, los cuales pueden estar asociados a la baja fluidez de este polvo en los ductos de la antorcha que lo transportan hacia la llama oxiacetilénica, lo que hace que el apilamiento de las partículas proyectadas sea discontinuo. Las fases presentes en este recubrimiento son $\mathrm{Al}_{2} \mathrm{O}_{3}-\alpha$ y $\mathrm{Al}_{2} \mathrm{O}_{3}-\gamma$, tal y como se presenta en los espectros de difracción de rayos $\mathrm{X}$ de la Figura 9 (b).

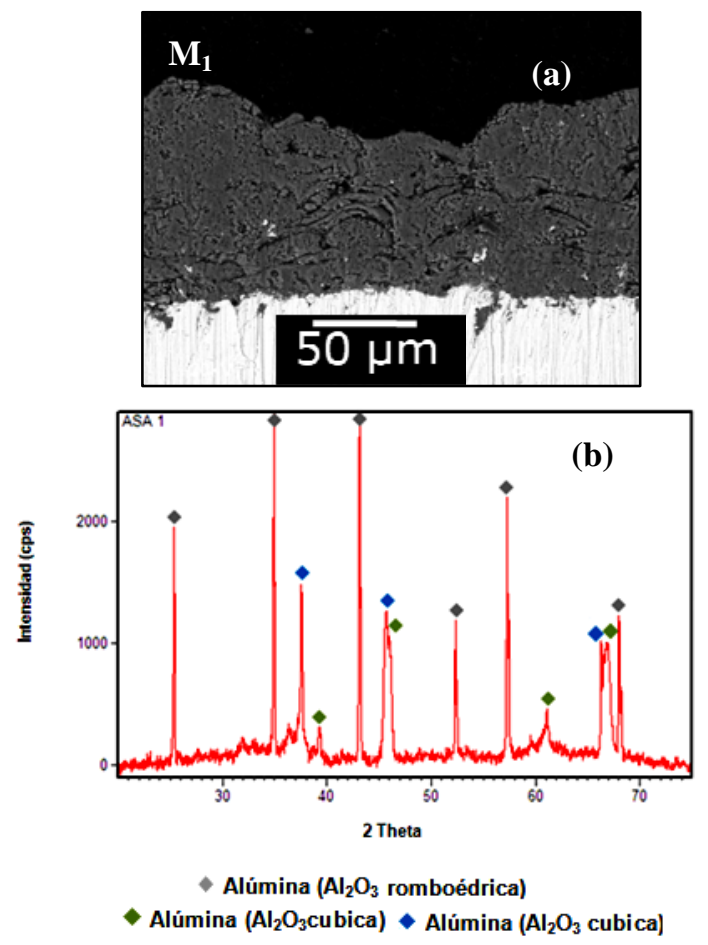

Figura 8: (a) Estructura del recubrimiento $\mathrm{M}_{1}$, (b) Espectro de difracción de Rayos X del recubrimiento $\mathrm{M}_{1}$. 
El recubrimiento elaborado a partir del catalizador $\mathrm{M}_{3}$ posee una estructura altamente cohesiva producto de la buena fusión de las partículas, Figura 10 (a). Además se observan poros intralamelares producto de la alta porosidad que posee el interior de las partículas utilizadas como materia prima. Los resultados del análisis de fases de este recubrimiento indican que es completamente amorfo, Figura 10 (b).

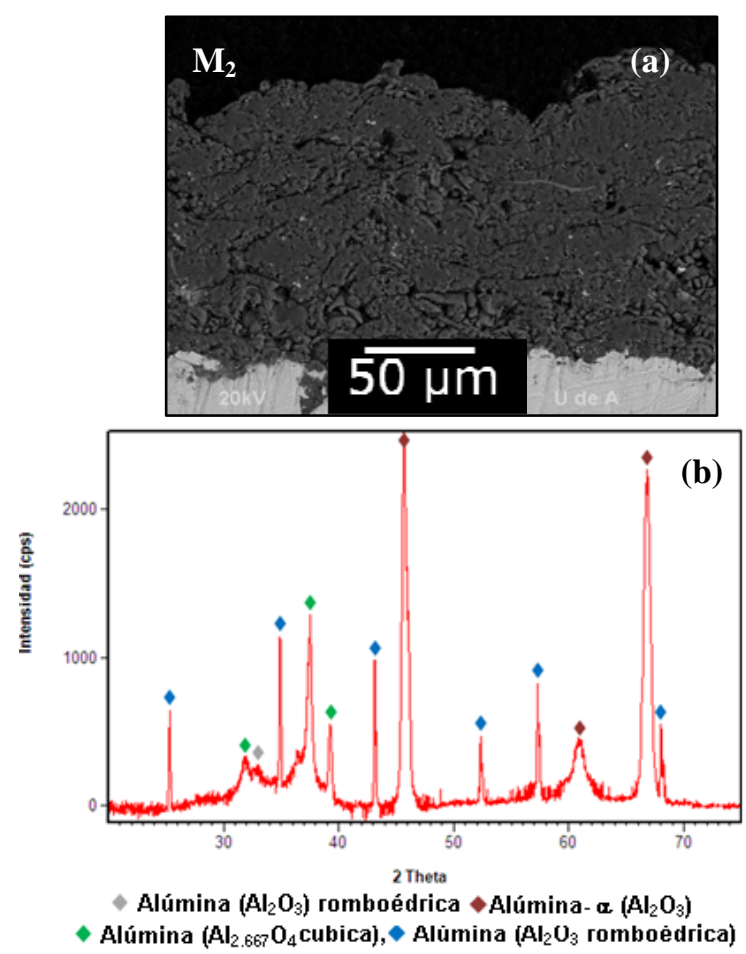

Figura 9: (a) Estructura del recubrimiento $\mathrm{M}_{2}$. (b) Espectro de difracción de Rayos $\mathrm{X}$ del recubrimiento $\mathrm{M}_{2}$
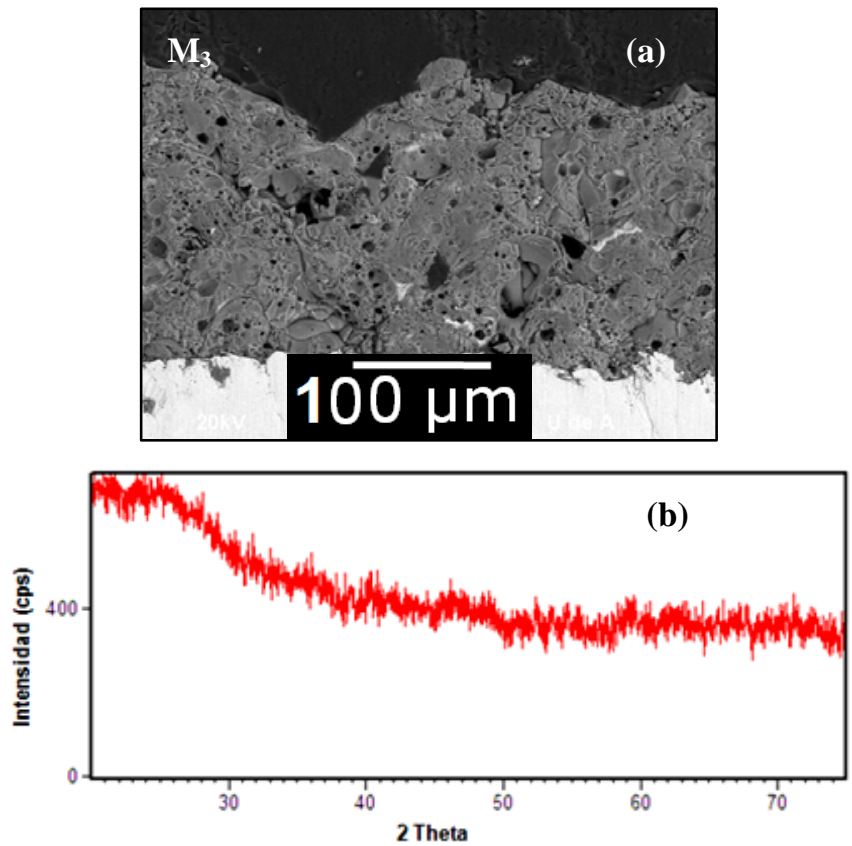

Figura 10: (a) Estructura del recubrimiento $\mathrm{M}_{3}$, (b) Espectro de difracción de Rayos X del recubrimiento $\mathrm{M}_{3}$. 
Los resultados de espesor, de las medidas de microdureza Vickers y de la adherencia de los recubrimientos obtenidos son mostrados en la Tabla 4.

Tabla 4: Resultados de la medida de dureza y de adherencia de los recubrimientos.

\begin{tabular}{|c|c|c|c|c|c|}
\hline \multirow[b]{2}{*}{ RECUBRIMIENTO } & \multirow[b]{2}{*}{$\begin{array}{l}\text { ESPESOR } \\
(\mu \mathrm{m})\end{array}$} & \multirow[b]{2}{*}{$\begin{array}{c}\text { DUREZA HV } \\
\text { [GPa] }\end{array}$} & \multicolumn{3}{|c|}{$\begin{array}{l}\text { RESULTADOS DE } \\
\text { ADHERENCIA }\end{array}$} \\
\hline & & & $\begin{array}{c}\text { ESFUERZO } \\
\text { DE } \\
\text { ADHESIÓN } \\
\text { [MPa] }\end{array}$ & $\begin{array}{c}\text { TIPO DE } \\
\text { FALLA }\end{array}$ & $\begin{array}{l}\% \text { DE } \\
\text { FALLA }\end{array}$ \\
\hline $\mathrm{M}_{1}$ & 99,9 & $\begin{array}{c}5,4 \pm 0,3 \\
(540 \pm 30 \mathrm{HV})\end{array}$ & $17,7 \pm 2,5$ & Adhesiva $^{*}$ & $<37$ \\
\hline $\mathrm{M}_{2}$ & 133,5 & $\begin{array}{c}8,0 \pm 1,5 \\
(800 \pm 150 \mathrm{HV}) \\
\end{array}$ & $2,9 \pm 0,8$ & Adhesiva $^{*}$ & 100 \\
\hline $\mathrm{M}_{3}$ & 266,6 & $\begin{array}{c}6,8 \pm 1,3 \\
(680 \pm 130 \mathrm{HV})\end{array}$ & $2,9 \pm 1,4$ & Adhesiva $^{*}$ & 100 \\
\hline
\end{tabular}

*La falla adhesiva hace referencia a que el desprendimiento se produce en la interfaz entre el sustrato y el recubrimiento.

La dureza de los recubrimientos de alúmina depositados por combustión oxiacetilénica normalmente varía entre 7 y $9 \mathrm{GPa}[8,13]$, por lo tanto el valor $(8 \pm 1,5 \mathrm{GPa})$ obtenido en el recubrimiento $\mathrm{M}_{2}$ es característico de este tipo de materiales. No obstante, la dureza del recubrimiento $\mathrm{M}_{1}$ fue considerablemente inferior, a pesar de estar constituido por las mismas fases que el $\mathrm{M}_{2}$, lo cual puede ser debido al efecto de borde impreso por la resina en la que fue montada la muestra y por el sustrato, dado el bajo espesor de este recubrimiento.

De otro lado, la dureza de los recubrimientos de Mullita, $3 \mathrm{Al}_{2} \mathrm{O}_{3}-2 \mathrm{SiO}_{2}$, normalmente son del orden de $5 \mathrm{GPa}$ [8], sin embargo, la dureza del recubrimiento $\mathrm{M}_{3}$ fue considerablemente superior, posiblemente debido a que los polvos utilizados para elaborar esta muestra poseen una cantidad superior de $\mathrm{Al}_{2} \mathrm{O}_{3}$, que aquellos polvos comerciales de alúmina-sílice.

El recubrimiento $\mathrm{M}_{1}$ presentó una excelente adherencia, con valores de esfuerzo de adhesión cercanos al del adhesivo utilizado en el ensayo, lo cual conlleva a que la falla se presente más por la adherencia del adhesivo que por el recubrimiento. Esto indica que si se hubiese utilizado un adhesivo de mayor resistencia, los valores de esfuerzo de adhesión hubiesen sido superiores a los obtenidos en la prueba.

Los esfuerzos de adhesión de los recubrimientos $\mathrm{M}_{2}$ y $\mathrm{M}_{3}$ son muy bajos, sin embargo, no se presentan fallas cohesivas, lo cual es reflejo de la buena cohesión entre las capas depositadas. Para mejorar la adherencia de estos recubrimientos se recomienda profundizar en la preparación superficial del sustrato o ser depositados sobre una capa base de níquel o de una aleación níquel-cobalto.

\section{CONCLUSIONES}

De acuerdo con la composición química, el tamaño y la forma de las partículas, los residuos de los catalizadores codificados como $\mathrm{M}_{1}, \mathrm{M}_{2}$ y $\mathrm{M}_{3}$, pueden ser utilizados como materia prima para la elaboración de recubrimientos mediante proyección térmica oxiacetilénica, para lo cual requieren de un tratamiento de clasificación mecánica del tamaño de partícula.

Los recubrimientos obtenidos a partir de los catalizadores $\mathrm{M}_{1}$ y $\mathrm{M}_{2}$ tratados están constituidos por $\mathrm{Al}_{2} \mathrm{O}_{3}-\alpha$ y $\mathrm{Al}_{2} \mathrm{O}_{3}-\gamma$, fases que confieren buenas propiedades mecánicas y buen desempeño tribológico, mientras que el recubrimiento de $\mathrm{Al}_{2} \mathrm{O}_{3}-\mathrm{SiO}_{2}$ obtenido a partir del catalizador $\mathrm{M}_{3}$ es amorfo y podría ser utilizado en aplicaciones donde se requiera de alta resistencia al choque térmico.

Los recubrimientos elaborados a partir de la muestra $\mathrm{M}_{1}$, tienen excelente adherencia sobre sustratos de acero al carbono. Sin embargo, se requiere aumentar su espesor, con el fin de que sean evidentes los valores de dureza característicos en recubrimientos constituidos por $\mathrm{Al}_{2} \mathrm{O}_{3}-\alpha$ y $\mathrm{Al}_{2} \mathrm{O}_{3}-\gamma$; mientras que los recubrimientos depositados a partir de las muestras $\mathrm{M}_{2}$ y $\mathrm{M}_{3}$ tienen excelente dureza. Sin embargo, se 
requiere trabajar en la modificación de la superficie del sustrato o deben ser depositados sobre una capa base para mejorar su adherencia.

\section{AGRADECIMIENTOS}

Los autores agradecen al Instituto Colombiano del Petróleo (ICP-ECOPETROL), por la co-financiación de este trabajo, realizado en el marco del convenio entre Ecopetrol y la Universidad de Antioquia 2007-01, así como a: los grupos de Investigación GIPIMME y GIMACYR de la Universidad de Antioquia y al Fondo de Apoyo Económico de la Facultad de Ingeniería por el apoyo logístico y económico brindado para adelantar el presente trabajo de investigación.

\section{BIBLIOGRAFIA}

[1] OZA, R., PATEL, S., "Recovery of Nickel from Spent Ni/ $\mathrm{Al}_{2} \mathrm{O}_{3}$ Catalysts using Acid Leaching, Chelation and Ultrasonication”, Research Journal of Recent Sciences, V. 1, pp. 1-3. 2012.

[2] SING, B. "Treatment of spent catalyst from the nitrogenous fertilizer industry-A review of the available methods of regeneration, recovery and disposal", Journal of Hazardous Materials, V. 167, pp. 2729. 2009 .

[3] RODRÍGUEZ, W.T., "Estudio de la remoción vía microbiana de $\mathrm{Ni}$ y $\mathrm{V}$ presentes en catalizadores gastados", M.Sc. Instituto Politécnico Nacional, Santiago de Querétaro, México, 2009.

[4] SABAJ, A. I.,"Efecto del soporte sobre la actividad y selectividad de catalizadores soportados de cobalto en la reacción de reformado de etanol con vapor", M.Sc. Universidad de Chile, Santiago de Chile, 2010.

[5] DAVID, S.J., PUJADO, P.R., Handbook of Petroleum Processing, Dordrecht, Springer, 2006.

[6] ROBERT, A.M., Handbook of Petroleum Refining Processes, 3 ed. MacGraw-Hill, 2004.

[7] JESUS, T.R.. Manual para la disposición de líquidos y sólidos en los laboratorios de la coordinación inspección de calidad de la gerencia complejo Barrancabermeja, Ing. Universidad Tecnológica de Pereira. Bucaramanga, Colombia, 2007.

[8] PAWLOSKY, L., The Science and Engineering of Thermal Spray Coatings, 2 ed. Villeneuve d'Ascq, John Wiley \& Sons, 2008.

[9] VARGAS, F., LATORRE, G., Recubrimientos Duros, En: Materiales de Ingeniería, V. 1, Capitulo 6, Bucaramanga, Colombia, Ecopetrol, 2007.

[10] TORRES, M., KHARISOV, I., NORA, V., "Recuperación de V y Mo de catalizadores gastados de la industria petroquímica. Parte I. Principios y Método de Recuperación”, Ciencia UNAL, V. 4, n. 2, pp. 194-208, 2001.

[11] ATKINSP. W., JONESL. L., Principios de Química. Los caminos del descubrimiento, 3 ed. S. L. Madrid, Medica Panamericana S.A, 2006.

[12] JAMES, H., Coatings and Coating Process for Metals, 1 ed. ASM International, 1998.

[13] HABIB, K.A., SAURA, J.J., FERRER, C., et al., "Comparison of flame sprayed $\mathrm{Al}_{2} \mathrm{O}_{3} / \mathrm{TiO}_{2}$ coatings: Their microstructure, mechanical properties and tribology behavior", Surface \& Coating Technology, V. 201, pp. 1436-1443, 2006. 